\title{
Papers
}

\section{D-dimer testing as an adjunct to ultrasonography in patients with clinically suspected deep vein thrombosis: prospective cohort study}

Enrico Bernardi, Paolo Prandoni, Anthonie W A Lensing, Giancarlo Agnelli, Giuliana Guazzaloca, Gianluigi Scannapieco, Franco Piovella, Fabio Verlato, Cristina Tomasi, Marco Moia, Luigi Scarano, Antonio Girolami on behalf of the Multicentre Italian D-dimer Ultrasound Study Investigators Group

\begin{abstract}
Objective To investigate the efficacy of using a rapid plasma D-dimer test as an adjunct to compression ultrasound for diagnosing clinically suspected deep vein thrombosis.

Design D-dimer concentrations were determined in all patients with a normal ultrasonogram at presentation. Repeat ultrasonography was performed 1 week later only in patients with abnormal D-dimer test results.
\end{abstract}

Main outcome measure Patients with normal ultrasonograms were not treated with anticoagulants and were followed for 3 months for thromboembolic complications.

Setting University research and affiliated centres. Subjects 946 patients with clinically suspected deep vein thrombosis.

Results Ultrasonograms were abnormal at presentation in $260(27.5 \%)$ patients. Of the remaining 686 patients tested for D-dimer, 88 (12.8\%) had abnormal concentrations. During follow up venous thromboembolic complications occurred in one of the 598 patients who were not treated with anticoagulants and who had an initial normal ultrasonogram and D-dimer concentration, whereas thromboembolic complications occurred in two of the 83 untreated patients who had abnormal D-dimer concentrations but a normal repeat ultrasonogram. The cumulative incidence of venous thromboembolic complications during follow up was $0.4 \%$ (95\% confidence interval $0 \%$ to $0.9 \%$ ). The rapid plasma D-dimer test used as an adjunct to compression ultrasonography resulted in a reduction in the mean number of repeat ultrasound examinations and additional hospital visits from 0.7 to 0.1 per patient. Conclusions Testing for $\mathrm{D}$-dimer as an adjunct to $\mathrm{a}$ normal baseline ultrasound examination decreased the number of subsequent ultrasound examinations considerably without any increased risk of venous thromboembolic complications in patients not receiving anticoagulants. The use of ultrasound and testing for D-dimer enabled treatment decisions to be made at the time of presentation in most patients.

\section{Introduction}

Compression ultrasound is widely used for the diagnostic management of patients with clinically suspected deep vein thrombosis. ${ }^{12}$ It has a high sensitivity for proximal vein thrombosis but is much less sensitive to thrombi confined to the calf veins. ${ }^{3}$ Some of these calf vein thromboses may quickly extend to the larger more proximal veins, presenting a high risk of pulmonary embolism. Therefore, repeat ultrasonography during a period of 1 week is indicated in all patients with a normal ultrasonogram at presentation. ${ }^{45} \mathrm{~A}$ single repeat ultrasound examination at 1 week can safely exclude deep vein thrombosis. ${ }^{6}$

D-dimer is a fragment specific to the degradation of fibrin. Several studies have shown its high sensitivity, moderate specificity, and high negative predictive value for suspected deep vein thrombosis. ${ }^{8-13}$ The high predictive value of a normal $\mathrm{D}$-dimer test result has potential in deciding which patients with a normal initial ultrasonogram should return for a repeat examination. We studied a large cohort of patients with clinically suspected deep vein thrombosis to determine whether the D-dimer test can be used as an adjunct to a normal ultrasonogram in these patients. Repeat ultrasonography was performed in patients with an abnormal D-dimer test result. The safety of withholding anticoagulant treatment in patients with normal ultrasonograms was evaluated during a 3 month follow up, with symptomatic venous thromboembolic complications as the outcome measure.

\section{Subjects and methods}

Patients

Consecutive outpatients with clinically suspected deep vein thrombosis of the leg were eligible for the study. Patients were excluded if they were pregnant, aged less than 18 years, had had a previous documented episode of venous thromboembolism, had concurrent signs or symptoms suggestive of pulmonary embolism, had received anticoagulant treatment for more than 48 hours before referral, needed anticoagulant treatment for indications other than venous disease, or were unable to return to a study centre for follow up because

\author{
Istituto di \\ Semeiotica Medica, \\ University of Padua \\ 35128 Padua, Italy \\ Enrico Bernardi, \\ research fellow \\ Luigi Scarano, \\ research fellow \\ Paolo Prandoni, \\ senior researcher \\ Antonio Girolami, \\ senior researcher \\ Azienda \\ Ospedaliera di \\ Padova Servizio di \\ Angiologia, \\ University of Padua \\ Fabio Verlato, \\ senior researcher \\ Istituto di Medicina \\ Interna e Vascolare, \\ Azienda \\ Ospedaliera di \\ Perugia, Policlinico \\ Monteluce, 06122 \\ Perugia, Italy \\ Giancarlo Agnelli, \\ senior researcher \\ Divisione di \\ Angiologia, \\ Azienda \\ Ospedaliera S \\ Orsola Malpighi, \\ 40138 Bologna, \\ Italy \\ Giuliana \\ Guazzaloca, \\ senior researcher \\ I Divisione Medica, \\ Ospedale Civile di \\ Venezia, Venice \\ 30100 , Italy \\ Gianluigi \\ Scannapieco, \\ senior researcher \\ continued over
}

BMJ 1998;317:1037-40 
Medicina Interna e Oncologia Medica, Policlinico San Matteo, Pavia Policlinico, Piazzale Golgi 2, 27100 Pavia, Italy

Franco Piovella, senior researcher

I Medicina, Ospedale di Bolzano, 39100 Bolzano, Italy Cristina Tomasi, senior researcher

Ospedale Maggiore e Università di

Milano, 20122 Milan, Italy

Marco Moia, senior researcher

Centre for Vascular Medicine, Academic Medical Centre, H-2, 1105 AZ Amsterdam,

Netherlands

Anthonie WA Lensing, senior researcher

Correspondence to: Dr Lensing a.w.lensing@ amc.uva.nl

Contributing members of the research group are given at the end of the paper of distance. Eligible patients gave their informed consent. The study protocol was approved by the institutional review boards.

\section{Study design}

We prospectively studied a large cohort of patients, with a 3 month follow up (fig). All patients had a compression ultrasound examination of the proximal veins. Patients with an abnormal ultrasonogram were treated with anticoagulants. A D-dimer test was perfomed in the remaining patients with a normal ultrasonogram. If the test results were normal, venous thrombosis was considered unlikely and follow up was arranged to record possible thromboembolic complications. If the test results were abnormal patients were still considered to be at risk of venous thrombosis, and repeat ultrasonography was scheduled for 1 week later. Only patients with an abnormal initial or repeat ultrasonogram received anticoagulant treatment. All patients who were followed up were told to return immediately if signs or symptoms suggestive of deep vein thrombosis or pulmonary embolism occurred. A follow up visit was scheduled at 3 months for all patients with normal ultrasonograms. At this visit they were questioned about their general health, recent hospital admissions, recurrence of signs and symptoms of venous thromboembolism, and anticoagulant treatment. Patients who were unable to attend the follow up sessions were visited at home or interviewed by telephone. During follow up, compression ultrasound examinations and D-dimer tests for possible venous thromboembolic complications were done as reported previously. ${ }^{7}$ A rapid enzyme linked immunosorbent assay (ELISA; Instant-IA D-dimer kit, Stago, Asnières, France) was used to determine the presence of D-dimers. Briefly, samples containing more than $0.5 \mu \mathrm{g} / \mathrm{ml} \mathrm{D}$-dimer produce a blue colour in the presence of nitroblue tetrazolium, the intensity of which reflects the concentration of D-dimer. D-dimer results were scored as normal (no colour development), abnormal, or inadequate for interpretation. The test has a high accuracy for detecting deep vein thrombosis with a sensitivity of more than $90 \%$, a specificity between 70 and $90 \%$, and a negative predictive value of more than $95 \% .{ }^{11-13}$ Reproducibility of the test is good ( $\kappa$ value 0.82 ).

\section{Statistical analysis}

We assumed that the high predictive value of a normal D-dimer test result in patients with a normal initial ultrasonogram would allow identification of a subset of patients at such a low risk of deep vein thrombosis that further ultrasonography could be safely excluded. Assuming an expected prevalence of 30\% for deep vein thrombosis we calculated that about 900 patients would be necessary to yield sufficiently narrow confidence intervals around the expected number of venous thromboembolic complications.

The total rate of venous thromboembolic complications was defined as either pulmonary embolism during the interval of baseline to repeat ultrasonography or pulmonary embolism or deep vein thrombosis during follow up. This was calculated using the Kaplan-Meier survival analysis. The complication rate was defined as one minus the complication free survival rate. This method takes into account the

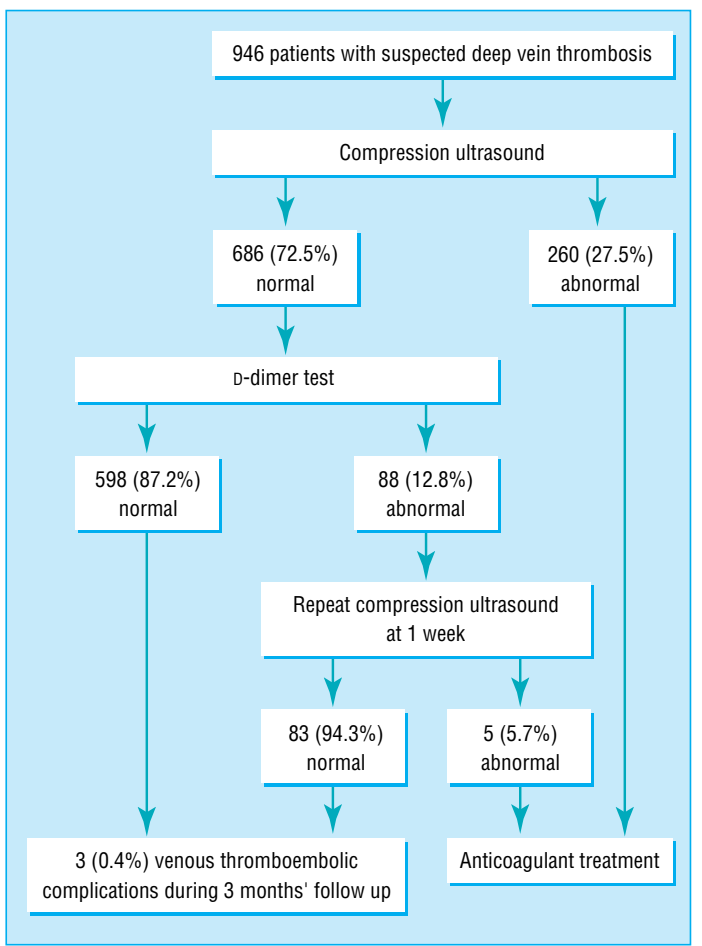

Compression ultrasonography and D-dimer evaluation in 946 patients with clinically suspected deep vein thrombosis

different numbers of patients at risk during the different time periods. An estimate of the exact 95\% confidence interval was made with the $\mathrm{SD}$ as calculated by the Kaplan-Meier analysis.

\section{Results}

\section{Patients}

Between September 1994 and February 1997 we considered 1142 consecutive outpatients with clinically suspected deep vein thrombosis. A total of 195 (17.1\%) patients were excluded: $93(47.7 \%)$ had a recurrent episode of venous thrombosis, 61 (31.3\%) were receiving long term anticoagulant treatment, $25(12.8 \%)$ were inaccessible geographically, 13 (6.7\%) had suspected pulmonary embolism at the time of referral, $2(1.0 \%)$ were aged less than 18 years, and $1(0.5 \%)$ was pregnant. One out of 947 possible participants refused to give informed consent, leaving 946 patients for inclusion in the study, of whom $587(62 \%)$ were female. The mean age of the patients was 59 years (range 20 to 93 years) and the mean interval between the onset of symptoms and the day of referral was 8 days (range 1 to 60 days).

\section{Venous thromboembolic complications}

Suitable ultrasonograms were obtained in all 946 patients. An abnormal ultrasonogram was present in $260(27.5 \%)$ patients at presentation, and anticoagulant treatment was initiated. All $686(72.5 \%)$ patients with a normal baseline ultrasonogram were tested for D-dimer; results were abnormal in 88 (12.8\%; including two inadequate results) of these patients. Four of the 598 untreated patients with an initial normal ultrasonogram and D-dimer test result died: two of cancer and one each of myocardial infarction and 
Characteristics of ultrasonographic strategies in patients with suspected deep vein thrombosis

\begin{tabular}{|c|c|c|c|c|c|}
\hline Reference & Diagnostic strategy & $\begin{array}{c}\text { No of } \\
\text { patients }\end{array}$ & $\begin{array}{l}\text { No }(\%) \text { with deep } \\
\text { vein thrombosis }\end{array}$ & $\begin{array}{l}\% \text { with venous } \\
\text { thromboembolic } \\
\text { complications } \\
(95 \% \mathrm{Cl})\end{array}$ & $\begin{array}{l}\text { Mean No of additional } \\
\text { ultrasound tests } \\
\text { (or venograms*) required per } \\
\text { initially referred patient }\end{array}$ \\
\hline Sluzewski et al ${ }^{5}$ & Ultrasonography at days 1,2 , and 7 & 118 & $32(27)$ & $1.3(0$ to 3.9$)$ & 1.3 \\
\hline Heijboer et al ${ }^{4}$ & Ultrasonography at days 1,2 , and 8 & 491 & $103(21)$ & $1.5(0.3$ to 2.8$)$ & 1.6 \\
\hline Cogo et al ${ }^{7}$ & Ultrasonography at days 1 and 7 & 1702 & $408(24)$ & 0.7 (0.3 to 1.2$)$ & 0.8 \\
\hline Birdwell et al ${ }^{6}$ & Ultrasonography at days 1 and 5-7 & 405 & $69(17)$ & $0.6(0.1$ to 2.1$)$ & 0.8 \\
\hline Wells et al ${ }^{14}$ & Ultrasonography at day 1 with clinical score $\dagger$ & 593 & $95(16)$ & 0.6 (0.1 to 1.8$)$ & $0.3^{*}$ \\
\hline Present study & $\begin{array}{l}\text { Ultrasonography at day } 1 \text {, if normal: } \mathrm{D} \text {-dimer; } \\
\text { repeat ultrasound at } 1 \text { week if } \mathrm{D} \text {-dimer positive }\end{array}$ & 946 & $265(28)$ & $0.4(0.0$ to 0.9$)$ & 0.1 \\
\hline
\end{tabular}

*Includes venography in $5.6 \%$ of patients.

†Low clinical score: normal ultrasonogram (stop), abnormal ultrasonogram (venography); moderate clinical score: normal ultrasonogram (repeat ultrasonography at 1 week), abnormal ultrasonogram (treatment); high clinical score: normal ultrasonogram (venography), abnormal ultrasonogram (treatment).

stroke. Two patients were lost to follow up after 1 and 2 months respectively. Four patients had suspected recurrent deep vein thrombosis, which was confirmed in one patient. This occurred 3 days after the normal baseline ultrasonogram and D-dimer test result. None of the 88 patients with an abnormal D-dimer test result and scheduled for repeat ultrasonography at 1 week developed pulmonary embolism in that period. Repeat ultrasonograms were abnormal in five patients, and anticoagulant treatment was started. The 83 patients with a normal repeat ultrasonogram did not receive anticoagulant treatment. None of these patients was lost to follow up, but one patient died of cancer. Three patients had suspected pulmonary embolism, which was confirmed in two of the patients after 2 and 3 weeks of follow up respectively. One of these patients died. Based on the normal results of the diagnostic investigation the cumulative incidence of venous thromboembolic complications during follow up in patients not treated with anticoagulants was $0.4 \%$ (95\% confidence interval $0 \%$ to $0.9 \%$ ). No further patients with suspected venous thromboembolic episodes were found at the follow up visit. However, anticoagulant treatment was started by other physicians in two cases despite normal ultrasonograms in these patients.

All 946 patients in our cohort had a baseline ultrasound examination, but only 88 needed repeat ultrasonography. In the remaining 598 patients a normal D-dimer test result avoided the need for repeat ultrasonography, reducing the mean number of repeat ultrasound examinations and extra hospital visits from 0.7 to 0.1 per patient.

\section{Discussion}

Compression ultrasound has considerably improved the management of clinically suspected deep vein thrombosis. However the need for a repeat examination at 1 week in patients with a normal baseline ultrasonogram is inconvenient and expensive as most of these patients do not have venous thrombosis. Our study shows that the D-dimer test is a useful adjunct to a normal ultrasonogram in patients with suspected deep vein thrombosis, and that this strategy simplifies the diagnostic investigation in such patients. Normal D-dimer test results were found in almost $90 \%$ of our patients with a normal initial ultrasonogram. We showed that this group of patients is at a low risk of venous thrombosis and that further ultrasonography and anticoagulant treatment could be safely excluded, as venous thromboembolic complications occurred in only one $(0.2 \%)$ patient during follow up. Repeat ultrasonography was needed in the 88 patients with a normal baseline ultrasonogram but an abnormal D-dimer test result as five patients were shown to have extended venous thrombosis at repeat ultrasonography 1 week later and two other patients had venous thromboembolic complications during follow up. Overall, the cumulative rate for venous thromboembolic complications during follow up for 3 months of all patients not treated with anticoagulants based on a normal ultrasonogram was $0.4 \%$; this is similar to earlier studies using serial compression ultrasound (table) ${ }^{4-7}{ }^{14}$ Fewer repeat ultrasound examinations is convenient for patients and cost effective. As only a small number $(5.7 \%)$ of our patients were identified with venous thrombosis by repeat ultrasonography 1 week later it can be questioned whether repeat examinations are necessary. An option is to manage all patients presenting with suspected deep vein thrombosis in one session on the day of referral. It should be realised, however, that the few patients who were identified by repeat ultrasonography had active progressive thrombosis and were clearly at risk of potentially fatal pulmonary embolism if undetected.

\section{D-dimer assays}

Various D-dimer assays have been introduced in the past decade. ${ }^{12}$ There are four basic methods for measuring D-dimer concentrations: (a) latex agglutination, (b) microplate ELISA, (c) immunofiltration (membrane ELISA), and (d) whole blood agglutination. Latex agglutination assays are relatively cheap and the results are quickly available, however they have a low sensitivity and specificity for venous thrombosis, which makes decisions on treatment difficult. The expensive microplate ELISAs consistently have the highest sensitivities of all the assays, but their role in venous thrombosis is limited by low specificity and high turnaround times. Two membrane ELISAs have been introduced (including the assay used in our study) that give results on single samples within minutes. These semiquantitative immunofiltration assays perform as well as the standard ELISAs but have better specificity. ${ }^{11-13}$ Our study shows the high accuracy of this D-dimer assay-that is, the small number of patients with a venous thromboembolic complication during follow up reflects the high sensitivity of the test whereas high specificity is implied by the small number of patients with an abnormal D-dimer test result but normal long 
Key messages

- Patients with clinically suspected deep vein thrombosis who have a normal baseline ultrasonogram have a small but clinically important risk of subsequent venous thromboembolic complications

- Repeat ultrasonography is not indicated in patients who have both a normal initial ultrasonogram and D-dimer test result, but should be performed at 1 week in those who have a normal initial ultrasonogram but an abnormal D-dimer test result

- D-dimer testing as an adjunct to a normal baseline ultrasonogram reduced the number of repeat ultrasound examinations considerably without an increased risk of venous thromboembolic complications in patients not receiving anticoagulants

- The ultrasonography and D-dimer testing strategy allowed treatment decisions to be made at the time of presentation in most patients

term outcomes. Clearly the higher the specificity of a D-dimer assay the fewer patients who should return for repeat ultrasonography. However, before D-dimer assays can be widely approved for the initial evaluation of venous thrombosis they need to be standardised. Clinical scoring is an alternative investigation for patients with suspected deep vein thrombosis. ${ }^{15}$ This concept was recently evaluated in a series of patients with suspected deep vein thrombosis: the patients did not have repeat ultrasonography if their clinical score showed a low probability of venous thrombosis. Repeat ultrasonography was, however, performed in those patients with a moderate probability of venous thrombosis, and venography was performed in those with a high probability. Follow up of patients not treated with anticoagulants showed that venous thromboembolic complications occurred infrequently $(0.6 \%$; table). The mean number of additional tests required per referred patient was 0.3 of which $15 \%$ was venography.

\section{Conclusion}

Testing for the presence of D-dimer after a normal baseline ultrasonogram in patients with suspected deep vein thrombosis reduces the number of repeat ultrasound examinations without an increased risk of venous thromboembolic complications during follow up of 3 months in patients not receiving anticoagulants. Ultrasonography in conjunction with D-dimer testing allowed treatment decisions to be made at the time of presentation in most patients.

The following investigators participated in the study: A Marchiori, L Rossi, C Sardella (Istituto di Semeiotica Medica, Padua, Italy); S Radicchia, F Sonaglia (Istituto di Medicina Interna e Vascolare, Perugia, Italy); G Palareti, C Legnani, S Coccheri (Divisione di Angiologia, Azienda Ospedaliera S Orsola Malpighi, Bologna, Italy); R Parisi, S Doria, C Bortoluzzi, C Slongo, G B Ambrosio (I Divisione Medica, Ospedale Civile di Venezia, Venice, Italy); M Barone, C Beltrametti, S Serafini, S Siragusa, E Ascari (Medicina Interna e Oncologia Medica, Policlinico S Matteo, Pavia, Italy); G Camporese, G P Signorini
(Servizio di Angiologia, Azienda Ospedaliera di Padova, Padua, Italy); N Tinkhauser, M Marchesi (I Medicina, Ospedale di Bolzano, Bolzano, Italy); and A Alatri, P Bucciarelli, and P M Mannucci (Ospedale Maggiore e Università di Milano, Milan, Italy).

Contributors: EB, PP, and AWAL designed the study protocol, initiated and coordinated the study, performed the analyses, interpreted the data, and were responsible for writing the paper: PP and AWAL will act as guarantors for the paper.FP, GA, GG, CT, GS, MM, FV, AG, and LS were the local principal investigators who discussed core ideas, participated in the protocol design and execution of the study, particularly data documentation and quality control, and edited the paper.

Funding: The study was supported in part by a grant from the Regione Veneto No 620/01/95 and by Boehringer Mannheim, Milan, Italy.

Conflict of interest: None.

1 Lensing AWA, Hirsh J, Büller HR. Diagnosis of venous thrombosis. In: Colman RW, Hirsh J, Marder VJ, Salzman EW, eds. Hemostasis and thrombosis: basic principles and clinical practice, 3rd ed. Philadelphia: JB Lippincott, 1993:1297-321.

2 Lensing AWA, Prandoni P, Brandjes D, Huisman PM, Vigo M, Tomasella $\mathrm{G}$, et al. Detection of deep-vein thrombosis by real-time B-mode ultrasonography. N Engl J Med 1989;320:342-5.

3 Kearon C, Julian JA, Newman TE, Ginsberg JS. Noninvasive diagnosis of deep venous thrombosis. Ann Intern Med 1998;128:663-77.

4 Heijboer H, Büller HR, Lensing AWA, Turpie AGG, Colly LP, Ten Cate JW. A comparison of real-time ultrasonography with impedance plethysmography for the diagnosis of deep-vein thrombosis in symptomatic outpatients. N Engl J Med 1993;329:1365-9.

5 Sluzewski M, Koopman MMW, Schuur KH, van Vroonhoven TJMV, Ruijs $\mathrm{JHJ}$. Influence of negative ultrasound findings on the management of inand outpatients with suspected deep-vein thrombosis. Eur J Radiol 1991;13:174-7.

6 Birdwell B, Raskob G, Whitsett T, Durica S, Comp P, George T, et al. Clinical validity of normal compression ultrasonography in outpatients suspected of having deep venous thrombosis. Ann Intern Med 1998; 128:1-7.

7 Cogo A, Lensing AWA, Koopman MMW, Piovella F, Siragusa S, Wells PS, et al. Compression ultrasound for diagnostic management of patients with clinically suspected venous thrombosis. BMJ 1998;316:17-20.

8 Becker DM, Philbrick JT, Bachhuber TL, Humphries JE. D-dimer testing and acute venous thromboembolism. Arch Intern Med 1996;156:939-46.

9 Bounameaux H, de Moerloose P, Perrier A, Reber G. Plasma measurement of D-dimer as diagnostic aid in suspected venous thromboembolism: an overview. Thromb Haemost 1994;71:1-6.

10 Wells PS, Brill-Edwards P, Stevens P, Panju A, Patel A, Douketis J, et al. A novel and rapid whole blood assay for D-dimer in patients with clinically suspected deep-vein thrombosis. Circulation 1995;91:2184-7.

11 Scarano L, Bernardi E, Prandoni P, Sardella C, Rossi L, Carraro P, et al. Accuracy of two newly described D-dimer tests in patients with suspected deep venous thrombosis. Thromb Res 1997;86:93-9.

12 Lee AYY, Ginsberg JS. The role of D-dimer in the diagnosis of venous thromboembolism. Curr Opinion Pulm Med 1997;3:275-9.

13 Legnani C, Pancani C, Palareti G, Guazzaloca G, Fortunato G, Grauso F, et al. Comparison of new rapid methods for D-dimer measurement to exclude deep vein thrombosis in symptomatic outpatients. Blood Coag Fibrinol 1997;8:296-302.

14 Wells PS, Anderson DR, Bormanis J, Guy F, Mitchell, Gray L, et al. Value of assessment of pretest probability of deep-vein thrombosis in clinical management. Lancet 1997;350:1795-8.

15 Wells PS, Hirsh J, Anderson DR, Lensing AWA, Foster G, Kearon C, et al. Accuracy of clinical assessment of deep-vein thrombosis. Lancet 1995;345:1326-30.

(Accepted 1 July 1998)

\section{Endpiece \\ Prescribing pressures}

Man has an inborn craving for medicines ... it is really one of the most serious difficulties with which we have to contend.

Sir William Osler, 1891

Submitted by Ann Dally, Wellcome Institute for the History of Medicine 